Document downloaded from:

http://hdl.handle.net/10251/139240

This paper must be cited as:

Gonzalez-Montoro, A.; González Martínez, AJ. (07-2). Performance comparison of largearea SiPM arrays suitable for

gamma ray detectors. Biomedical Physics \& Engineering Express. 5(4):1-10.

https://doi.org/10.1088/2057-1976/ab0f6e

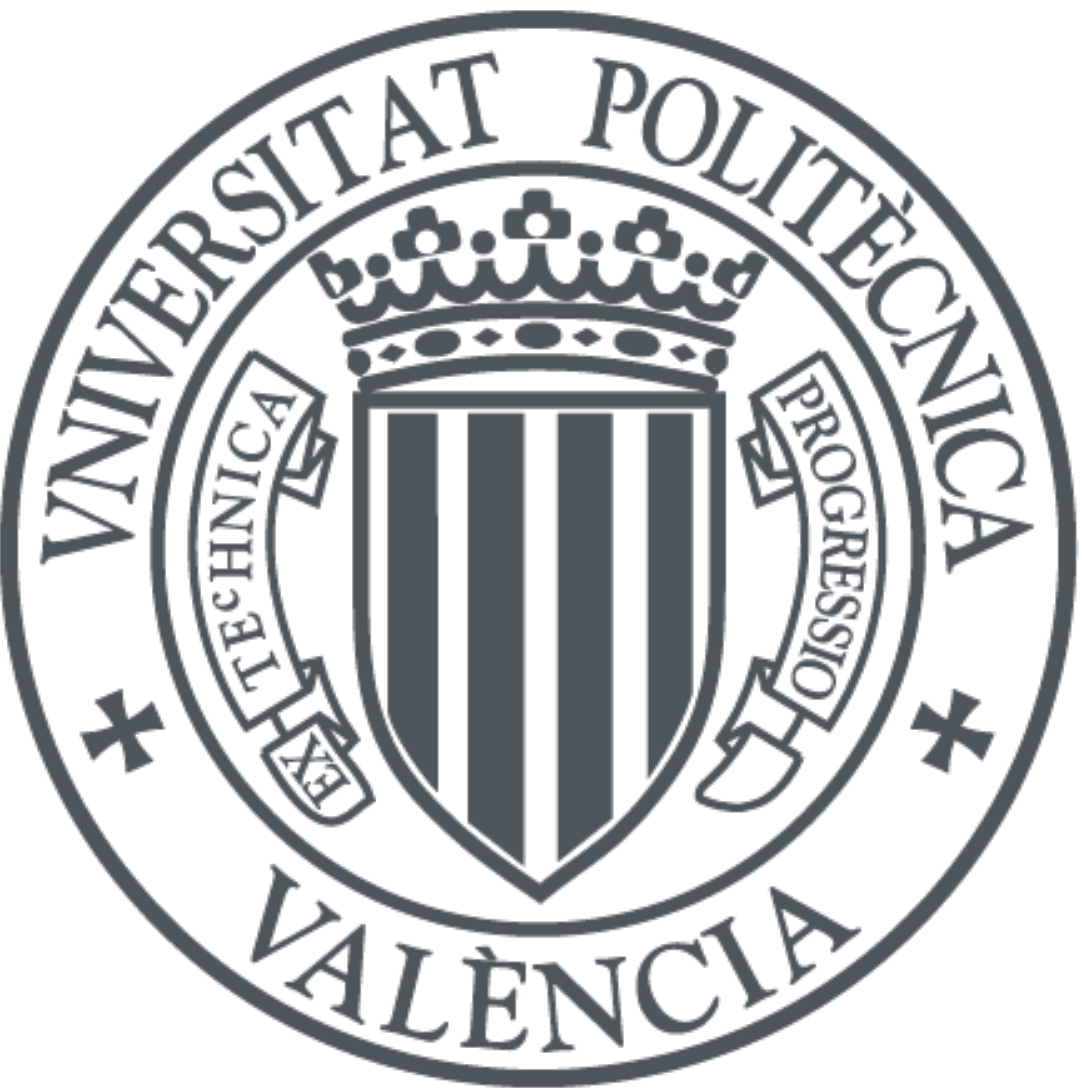

The final publication is available at

https://doi.org/10.1088/2057-1976/ab0f6e

Copyright IOP Publishing

Additional Information 


\title{
Performance Comparison of Large-Area SiPM Arrays Suitable for Gamma Ray Detectors
}

\author{
Andrea Gonzalez-Montoro ${ }^{1}$ and Antonio J. Gonzalez ${ }^{1}$ \\ ${ }^{1}$ Instituto de Instrumentación para Imagen Molecular (I3M), CSIC — Universitat Politècnica de \\ València, 46022 Valencia, Spain \\ E-mail: agonzalez@i3m.upv.es
}

\begin{abstract}
The use of photosensors of the type Silicon Photomultpliers (SiPM) has widely been extended in recent years for multiple applications in both research and industry. However, there is a lack of comparative studies of different SiPMs under the same conditions, making it difficult to choose the most appropriate one for a specific application. SiPM arrays are suitable for gamma rays detectors, especially when covering large active areas. They are used either in clinical or pre-clinical scenarios, constituting Single-Photon Emission Computed Tomography (SPECT) and Positron Emission Tomography (PET) scanners, or just gamma cameras.

The current work presents, for the first time, a comparative study between SensL, Hamamatsu Photonics and KETEK arrays of $12 \times 12$ SiPMs, with individual active areas of $3 \mathrm{~mm} \times 3 \mathrm{~mm}$, thus covering a total active area of about $5 \mathrm{~cm} \times 5 \mathrm{~cm}$. In this study, we have first evaluated their bias and temperature dependencies, resulting in a very similar behaviour with just a slightly larger dependency of the tested Hamamatsu parts. We also report the performance of detector blocks based on these three SiPM arrays, when coupled to pixelated crystal arrays and monolithic scintillators, in terms of photon impact estimation accuracy, as well as energy resolution. In the case of the monolithic crystals, also the depth of interaction resolution was determined. Both monolithic and pixelated blocks are nowadays widely used in academia or are commercially available for molecular imaging systems.

The results obtained for the three SiPM arrays when using those crystals, are comparable, without observing any significant different among them.
\end{abstract}

Keywords: Silicon Photomultipliers (SiPM), Gamma ray detector, Positron Emission Tomography (PET), scintillation crystal

\section{Introduction}

In the last 10-15 years there have been significant efforts in the use of photosensors based on solid state technology such as Silicon Photomultipliers (SiPMs). These types of photosensors are used in a wide field of applications, such as high energy physics, light detection and ranging (LiDAR) or in gamma ray detectors which are used in Positron Emission Tomography (PET), Single Photon Emission Computed Tomography (SPECT) or simply gamma cameras [1], [2]. In contrast to the former technology, the so-called Photomultiplier Tubes (PMTs) for which there were just few providers, SiPMs are more widely available. The advantages and disadvantages of SiPMs versus PMTs have been widely described elsewhere [3]-[5]. Shortly, in addition to the broader availability, other attractive advantages of SiPMs are their compatibility to work immersed in strong magnetic fields, their compactness and the low operation bias conditions. However, in contrast to PMTs, SiPMs suffer from dark counts (DC) since they are based on the solid state technology. SiPMs require therefore working in temperature controlled environments.

SiPMs are the evolution from their analogue type of photosensor named Avalanche Photodiode (APD). The main difference between those two photosensors is that APDs 
work in the avalanche regime, whereas SiPMs in the Geiger regime [6]. SiPMs make it possible to achieve significant higher gains $\left(10^{6}\right.$, similar to PMTs) than APDs $\left(10^{2}-10^{3}\right)$, with overall improved performance [7].

There has been also an evolution in performance when referring to SiPMs. The characteristic that has been improved more is the reduction of the DC, which negatively affects their performance, especially when referring to gamma ray detectors, as it is the scope of this work. Also, more stable SiPMs regarding bias and temperature variations are currently available. SiPMs with higher efficiencies at different wavelengths beyond the visible range also exist [8], [9].

Some SiPM providers offer single SiPMs for a variety of active areas, ranging from $1 \mathrm{~mm} \times 1 \mathrm{~mm}$ to typically $6 \mathrm{~mm} \times$ $6 \mathrm{~mm}$. Nowadays, the most used SiPM package type is the so-called through-silicon-vias (TSV), in contrast to somehow former wire bonding approaches. TSV provides a better filling factor for the configuration of SiPM arrays. There are some SiPM arrays directly available from providers. Standard arrays configurations are $4 \times 4,8 \times 8$, or $12 \times 12$ SiPMs. However, one can find other arrangements, such as linear arrays (for instance $1 \times 16$ ), or build custom ones. SiPMs with active areas different than squares are also exist.

We have evaluated detectors blocks for clinical [10], [11] and pre-clinical PET [12], [13] imaging with different scintillators and photosensor technology, with special focus on the use of monolithic crystals. In this work we present a comparative study between arrays of 144 SiPMs with $3 \mathrm{~mm}$ $\times 3 \mathrm{~mm}$ active area each in a $12 \times 12$ configuration, leading to an approximate total coverage of $5 \mathrm{~cm} \times 5 \mathrm{~cm}$. We have tested arrays from three providers namely ONSemiconductor (SensL Division) [14], Hamamatsu Photonics (Hamamatsu, Japan) [15] and KETEK (Munich, Germany) [16].

\section{Materials and methods}

In this work, three arrays of SiPMs from different companies had been evaluated and their performance is shown in a comparative way. Two types of scintillation crystals have been used: i) a $15 \mathrm{~mm}$ thick LYSO monolithic block and, ii) a pixelated crystal array with $1.5 \mathrm{~mm}$ pitch size.

\subsection{SiPM arrays}

The SiPMs used in SensL's configuration are of the type C-Series with wire bonding package (non-TSV) [14], and directly obtained as a commercial item. Hamamatsu (S13360) [18] and KETEK (PM3325-WB) [19] parts are TSV, and were explicitly built for this study. The SiPM pitch (center-to-center active area distance) for the SensL and KETEK arrays is $4.2 \mathrm{~mm}$ and $4.25 \mathrm{~mm}$ for Hamamatsu arrays. All arrays were assembled in a similar Printed Circuit
Board (PCB), with four connectors on the backside providing output signals for all 144 SiPMs. The main specifications of the used SiPMs are summarized in Table 1. All SiPMs present similar overall performances, as described by the manufactures. Hamamatsu parts have a breakdown voltage slightly higher than others, about $50 \mathrm{~V}$, also exhibiting a slightly higher temperature dependency and DC rates. Microcell sizes vary from $25 \mu \mathrm{m}$ to $50 \mu \mathrm{m}$ among the different tested SiPMs. These values are important for certain applications but do not play an important role in the results that we present here. Photographs of the three arrays are shown in Figure 1.

\begin{tabular}{|c|c|c|c|}
\hline & SensL & Hamamatsu & KETEK \\
\hline Microcell $(\mu \mathrm{m})$ & 35 & 50 & 25 \\
\hline $\begin{array}{c}\text { Breakdown } \\
\text { voltage }\left(\mathrm{V}_{\mathrm{br}}\right)\end{array}$ & $24.2-24.7$ & $53 \pm 5$ & 25.7 \\
\hline $\begin{array}{c}\text { Temperature } \\
\text { dependency of } \\
\mathrm{V}_{\mathrm{br}} \mathrm{mV} /{ }^{\circ} \mathrm{C} \\
\end{array}$ & 21.5 & 54.0 & 18.0 \\
\hline $\begin{array}{c}\text { Temperature } \\
\text { dependency of } \\
\text { gain } \% /{ }^{\circ} \mathrm{C} \\
\end{array}$ & 0.8 & 2 & $0.5 @ 5 \mathrm{~V}_{\mathrm{ov}}$ \\
\hline $\begin{array}{c}\text { Photon Detection } \\
\text { Efficiency \% @ } \\
430 \mathrm{~nm} \\
\end{array}$ & $\begin{array}{c}31 \% @ 2.5 \mathrm{~V}_{\mathrm{ov}} \\
41 \% @ 5 \mathrm{~V}_{\mathrm{ov}}\end{array}$ & $40 \% @ 3 \mathrm{~V}_{\mathrm{ov}}$ & $\begin{array}{c}31 \% @ 2.5 \mathrm{~V}_{\mathrm{ov}} \\
43 \% @ 5 \mathrm{~V}_{\mathrm{ov}}\end{array}$ \\
\hline $\begin{array}{c}\text { Dark Count Rate } \\
\mathrm{kHz} / \mathrm{mm}^{2}\end{array}$ & $\begin{array}{l}100-287 \\
\text { (a) } 2.5 \mathrm{~V}_{\mathrm{ov}}\end{array}$ & $\begin{array}{c}500-1500 \\
\text { (a) } 3 \mathrm{~V}_{\mathrm{ov}} \\
\end{array}$ & $400 @ 5 \mathrm{~V}_{\mathrm{ov}}$ \\
\hline Gain & $\begin{array}{l}3 \times 10^{6} \\
\text { (a) } 2.5 \mathrm{~V}_{\mathrm{ov}}\end{array}$ & $\begin{array}{c}1.7 \times 10^{6} \\
\text { @ } 3 \mathrm{~V}_{\mathrm{ov}}\end{array}$ & $\begin{array}{c}0.87 \times 10^{6} \\
@ 2.5 \mathrm{~V}_{\mathrm{ov}} \\
1.74 \times 10^{6} @ 5 \mathrm{~V}_{\mathrm{ov}}\end{array}$ \\
\hline
\end{tabular}

Table 1. Main specifications of the three SiPMs elements used in the photosensors arrays. $\mathrm{V}_{\mathrm{br}}$ stands for breakdown voltage, $\mathrm{V}_{\mathrm{ov}}$ stands for over voltage.
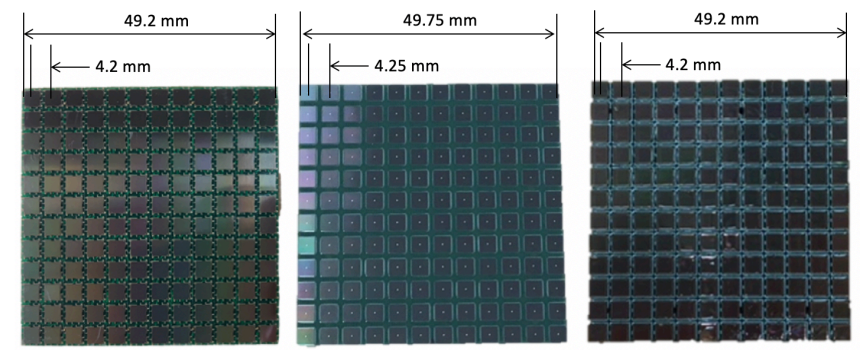

Figure 1. Photographs of the three SiPM arrays. From left to right, SensL, Hamamatsu and KETEK configurations. The pitch sizes and overall active areas are shown above the particular SiPM arrays.

\subsection{Scintillation crystals}

In gamma ray detection for Gamma Cameras, SPECT or PET, both pixelated and monolithic crystals are used. Therefore, for the evaluation of the three SiPMs arrays a LYSO pixaleted scintillation array and a LYSO monolithic crystal has been used. The use of the two crystal types provides complementary information. The same crystals were assembled with each one of the 3 SiPM tested arrays.

The monolithic scintillation crystal has dimensions of 50 $\mathrm{mm} \times 50 \mathrm{~mm} \times 15 \mathrm{~mm}$. While all faces are polished, the 
lateral faces $(50 \mathrm{~mm} \times 15 \mathrm{~mm})$ are black painted, and the entrance face includes a retro-reflector (RR) layer [20]. The scintillation crystal array has $33 \times 33$ pixels of $1.5 \mathrm{~mm}$ pitch and $6 \mathrm{~mm}$ height. All pixel faces are as-cut (rough) except the exit one that is polished and in contact with the photosensor array. Enhance specular reflectors (ESR from $3 \mathrm{M}$, about $70 \mu \mathrm{m}$ thickness) are used in all rough faces. A diffusor layer of $1.12 \mathrm{~mm}$ thickness was added between the pixelated crystal and the photosensor. All optical elements were coupled using optical grease of the type BC630 (Saint Gobain) [21].

\subsection{Readout electronics and $D A Q$}

In this work we have made use of analog readout electronics that extract signals from each SiPM row and column [20]. It has been earlier demonstrated that this method permits accurate detector spatial resolution measurement both for pixelated or monolithic crystals [22]. With the current SiPM arrays configurations of 12 rows and 12 columns, 24 signals are read out, amplified and sent to the data acquisition system (DAQ). That DAQ system looks for coincidences in a $5 \mathrm{~ns}$ window, and digitizes the 24 signals belonging to each detector block using charge integrators (250 ns) with 12 bit precision [10], [13].

The gamma ray impact position is obtained using the 12 row and 12 column signals by applying centre of gravity approaches $(\mathrm{CoG})$. In order to improve the detectability at the edges of the detector blocks, the digitized signals have been risen to the power of two prior to CoG calculation [23]. When using monolithic crystals, we are also providing comparative information regarding the photon depth of interaction (DOI) accuracy. DOI is determined using the figure of merit defined as the average ratio of the energy and maximum collected signal for each row and column projection [20].

All measurements were taken at a controlled temperature environment of around $18^{\circ} \mathrm{C}$.

\subsection{Bias and temperature dependency}

The SiPM performance regarding bias voltage and temperature dependency have been evaluated using the monolithic scintillation crystals, since the readout and DAQ electronics are better suited for these crystals, due to amplification reasons. Nevertheless, we also collected data for several bias voltages when coupling the photosensor arrays to a pixelated crystal matrix.

DC in SiPM increases with temperature and bias voltage. We have first studied the bias voltage applied to the different SiPM arrays. We scanned the bias in steps of $1 \mathrm{~V}$ (and $0.5 \mathrm{~V}$ around the best observed performance) from their breakdown voltage $\mathrm{V}_{\text {br }}$ up to about $5 \mathrm{~V}$ over this $\mathrm{V}_{\text {br }}$ (Table 2).
For these evaluation tests, a small size ${ }^{22} \mathrm{Na}$ source $(0.25$ $\mathrm{mm}$ in diameter) with about $20 \mu \mathrm{Ci}$ activity was placed in front of the detector under evaluation for 15 seconds $(3-4 \mathrm{~cm}$ away from the entrance crystal face). We measured in single mode, meaning that all the events were collected and not only the coincidences. Single measurements allowed us to provide feedback on the linearity response by observing the $511 \mathrm{keV}$ annihilation peak and the single gamma ray emission at $1275 \mathrm{keV}$, when using the ${ }^{22} \mathrm{Na}$ source. We made sure that pile-up events were not presented, and that electronic or DC noise did not affect the results, by observing the detector signals and the energy spectra.

\begin{tabular}{c|c|c} 
& $\mathrm{V}_{\mathrm{br}}(\mathrm{V})$ & Scanning Range $(\mathrm{V})$ \\
\hline SensL & $24.2-24.7$ & $26-32$ \\
\hline Hamamatsu & $53 \pm 5$ & $53-58$ \\
\hline KETEK & 25.7 & $28-33$
\end{tabular}

Table 2. Breakdown voltage and voltage scanning ranges selected for each tested SiPM array.

\subsection{Energy resolution and position determination}

We have evaluated the detector blocks (SiPMs, readout and scintillation crystals) in terms of spatial, energy and depth of interaction resolution. When using the monolithic blocks, the measurements were carried out in coincidence mode, using the optimum bias voltage found in the bias dependency experiments. For this purpose, a reference detector based on an identical $15 \mathrm{~mm}$ thick monolithic crystal was used. An array of $11 \times 11^{22} \mathrm{Na}$ sources with 4.6 $\mathrm{mm}$ pitch and $1 \mathrm{~mm}$ in diameter (total activity about $20 \mu \mathrm{Ci}$ ) was placed in front of a tungsten collimator, that was in between this source array and detector block under study. The collimator has a thickness of $24 \mathrm{~mm}$ and drilled holes of $1.2 \mathrm{~mm}$ in diameter at the sources position. We acquired data for about 4 hours with each detector block. We applied a software collimation to the data with a slope of $2.1^{\circ}$ from the normal [24]. This means that lines of response with large angles are rejected, helping at improving the signal to noise ratio.

We have analysed the central row and column for each array acquisition. We have determined the spatial resolution as the measured average full width at half of the maximum $(<$ FWHM $>$ ) through multi-Gaussian fitting. Thus, the source size contribution has not been removed from the results.

The energy resolution was evaluated for each source along one diagonal, including those at the corners.

The DOI performance is obtained by performing lateral incidence beam experiments. A ${ }^{22} \mathrm{Na}$ source was mechanically collimated using a tungsten block of $60 \mathrm{~mm}$ in diameter and $30 \mathrm{~mm}$ height with a $2 \mathrm{~mm}$ drilled hole. The source and collimator arrangement were positioned at different impinging heights on the detector block under test, namely $2.5 \mathrm{~mm}, 7.5 \mathrm{~mm}$ and $12.5 \mathrm{~mm}$ from the photosensor. Data were acquired for 20 minutes and a ROI of $5 \mathrm{~mm} \times 5$ 
$\mathrm{mm}$ centred at $7 \mathrm{~mm}$ from the crystal edge has been selected (see similar experimental details in [24]). The average FWHM of the DOI was deduced after calibration into millimetres [20], [24].

When analysing the spatial and DOI resolution, an additional energy filter for the range of $430-590 \mathrm{keV}$ had been applied.

When using the pixelated crystal array, the spatial resolution was evaluated using the peak-to-valley $(\mathrm{P} / \mathrm{V})$ of the pixels as a function of the applied voltages for the three SiPMs array cases. We selected one row and one column of pixels and estimated the ratio between the average maximum values and its baseline. We evaluated the energy resolution for the central pixel.

\section{Results}

\subsection{Bias and temperature dependency}

We analysed the photopeak position (in channels) and obtained the energy resolution (FWHM/Centroid) as a function of the applied voltage for a region of interest (ROI) of approximately $8 \mathrm{~mm} \times 8 \mathrm{~mm}$ at the crystal centre. We observed a gain shift when increasing the bias voltage, as expected, since the SiPM photon detection efficiency (PDE) improves (Figure 2 top). PDE is a function of the incident light wavelength, the applied overvoltage and the microcell fill factor (structure of the sensor). We found similar performance with the bias variation for the three arrays. Regarding energy resolution, best values at about 13-15\% were obtained at a bias voltage of $29.5,56.5$ and $33 \mathrm{~V}$, for SensL, Hamamatsu, and KETEK devices, respectively (Figure 2 bottom).

Using the same detector configuration, we have also studied the system dependency with the temperature. We scanned the three blocks for a temperature range of $13-38{ }^{\circ} \mathrm{C}$, in steps of $1{ }^{\circ} \mathrm{C}$. The SiPMs arrays were biased at the optimum voltage as found above. The photopeak variation for the three SiPM arrays as a function of the temperature is illustrated at the top of Figure 3. We determined gain variations in $\left[\% /{ }^{\circ} \mathrm{C}\right]$ units of $0.6 \pm 0.1,2.7 \pm 0.1$ and $0.9 \pm 0.1$ for the SensL, Hamamatsu, and KETEK parts, respectively.

Energy resolution dependence with temperature is shown at the bottom of Figure 3. A slightly degradation is observed when increasing the temperature. Since we used the ${ }^{22} \mathrm{Na}$ source, we also studied the detector blocks linearity responses through the ratio of the $1275 \mathrm{keV}$ and $511 \mathrm{keV}$ peaks (theoretical value equal to 2.49). Table 3 summarizes the average peak ratios, energy resolution at $25{ }^{\circ} \mathrm{C}$, and energy resolution variation in $\% /{ }^{\circ} \mathrm{C}$ (estimated from the slope of the curves shown in this figure panel), measured for each case. The error bars were calculated as the standard deviation of the measured values.

\begin{tabular}{c|c|c|c} 
& Peak ratio & $\begin{array}{c}\text { Energy res } \\
(\%) \text { at } 25^{\circ} \mathrm{C}\end{array}$ & $\begin{array}{c}\text { Variation } \\
\left(\% /{ }^{\circ} \mathrm{C}\right)\end{array}$ \\
\hline SensL & $2.5 \pm 0.1$ & $13.7 \pm 0.2$ & $0.02 \pm 0.01$ \\
\hline Hamamatsu & $2.5 \pm 0.1$ & $13.5 \pm 0.3$ & $0.03 \pm 0.01$ \\
\hline KETEK & $2.5 \pm 0.1$ & $14.6 \pm 0.2$ & $0.07 \pm 0.01$
\end{tabular}

Table 3. Average energy peak ratio, energy resolution at $25{ }^{\circ} \mathrm{C}$ and energy resolution variation $\left(\% /{ }^{\circ} \mathrm{C}\right)$, for each tested SiPM.

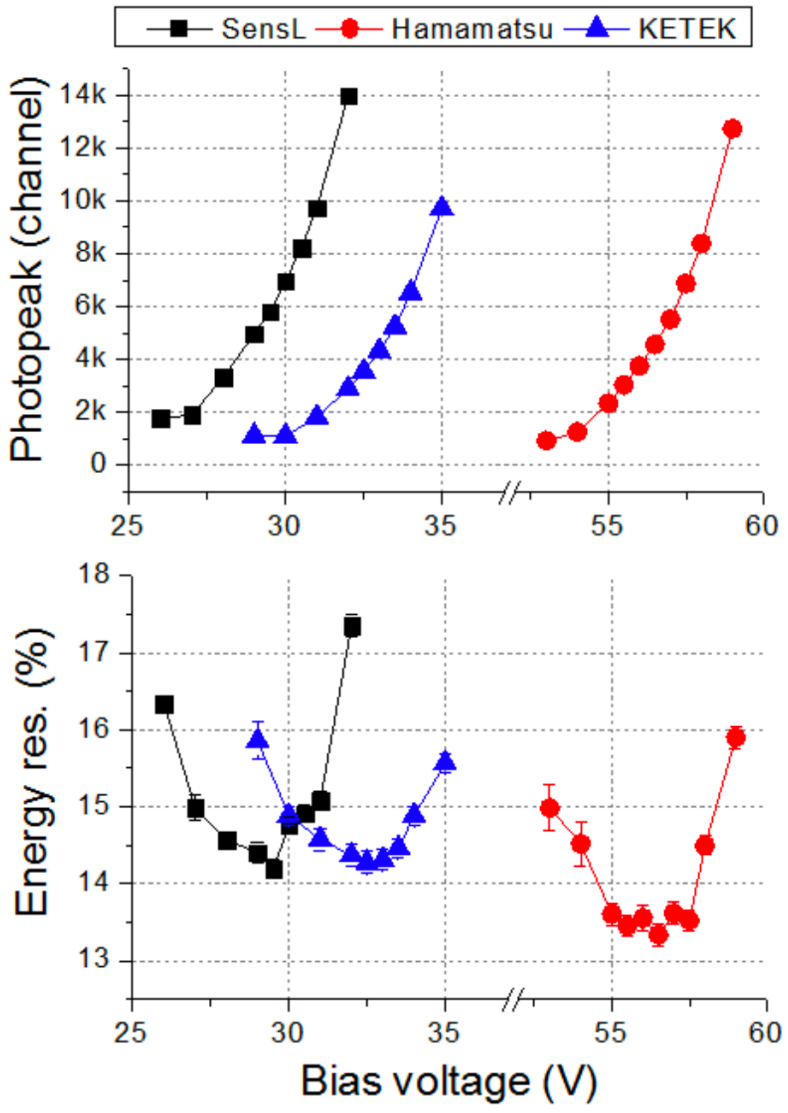

Figure 2. Photopeak (in channels) and energy resolution as a function of the bias voltage for the three SiPM arrays.
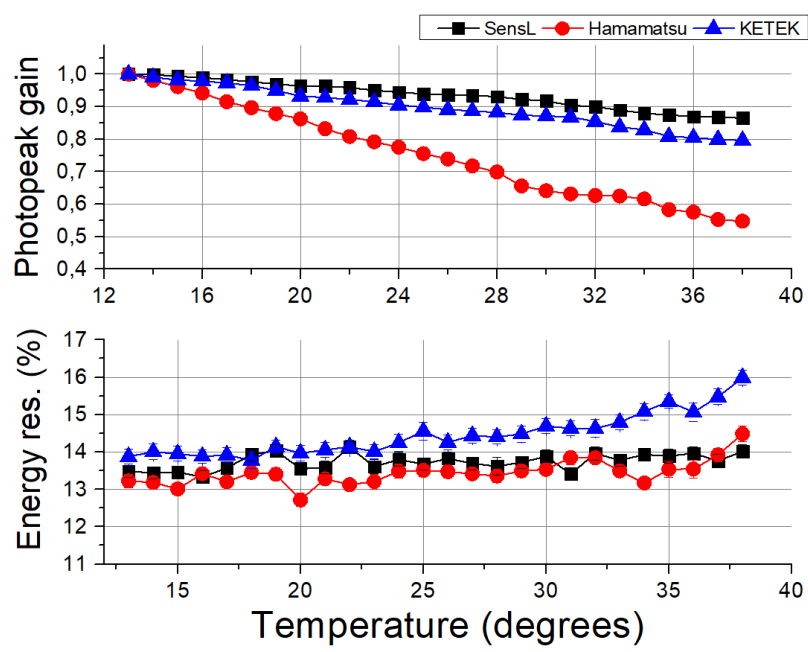

Figure 3. Photopeak gain and energy resolution plots as a function of the temperature for the three SiPM arrays. 


\subsection{Monolithic based detectors performance}

The flood image after irradiation with a uniform source is illustrated in the top-left of Figure 4, for the Hamamatsu SiPM array case. Top-right of the same figure shows the flood image when the collimated sources array was used, also applying the software collimation. The bottom-left panel of the figure shows an example of the flood image obtained after lateral incidence to the crystal. An energy spectrum is also shown in this figure at the bottom-right panel.
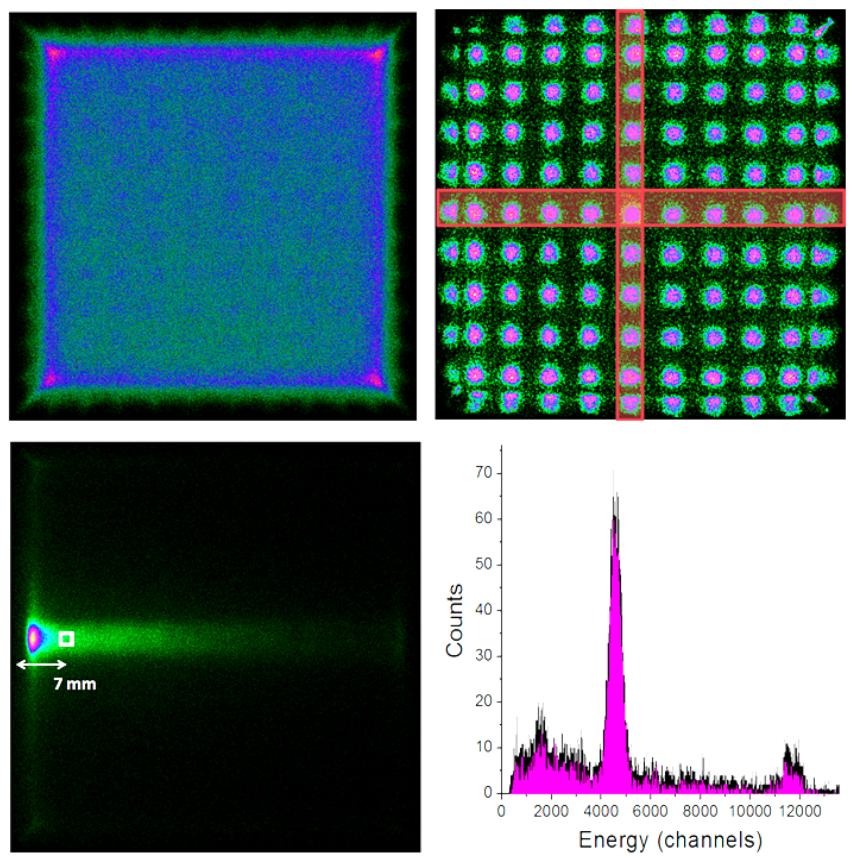

Figure 4. Top-left, flood map obtained with the monolithic crystal and the Hamamatsu SiPM array using uniform radiation. Top-right, flood map for the same detector configuration when the collimated array of sources is used. Bottom-left, flood map when lateral incidence beam to the crystal is used. Bottom-right, energy profile but when working in single mode during the bias dependency tests.

In Figure 5 top, we have plotted the measured spatial resolutions. When calculating the $\langle\mathrm{FWHM}\rangle$, we obtained values of $1.9 \pm 0.3 \mathrm{~mm}, 1.9 \pm 0.1 \mathrm{~mm}$ and $1.8 \pm 0.3 \mathrm{~mm}$, for the SensL, Hamamatsu and KETEK SiPM arrays, respectively. The error bars are again calculated as the standard deviation of all data points.

Regarding energy resolution results, the data at the edges of the crystal exhibit certain light truncation in a monolithic black painted block, providing poorer performance. The three data sets follow the same behaviour. The average calculated values are $12.9 \pm 1.6 \%, 13.3 \pm 1.3$ and $12.8 \pm 1.6 \%$, for SensL, Hamamatsu and KETEK, respectively (bottom of Figure 5).

The DOI resolution resulted in values of $3.2 \pm 0.3 \mathrm{~mm}$, $3.1 \pm 0.6 \mathrm{~mm}$ and $3.2 \pm 0.7 \mathrm{~mm}$, for SensL, Hamamatsu and KETEK, respectively. Figure 6 shows the obtained DOI histograms for the three lateral beam positions, overlapping with the distribution obtained when normally impinging the crystal and considering data in the same image ROI.

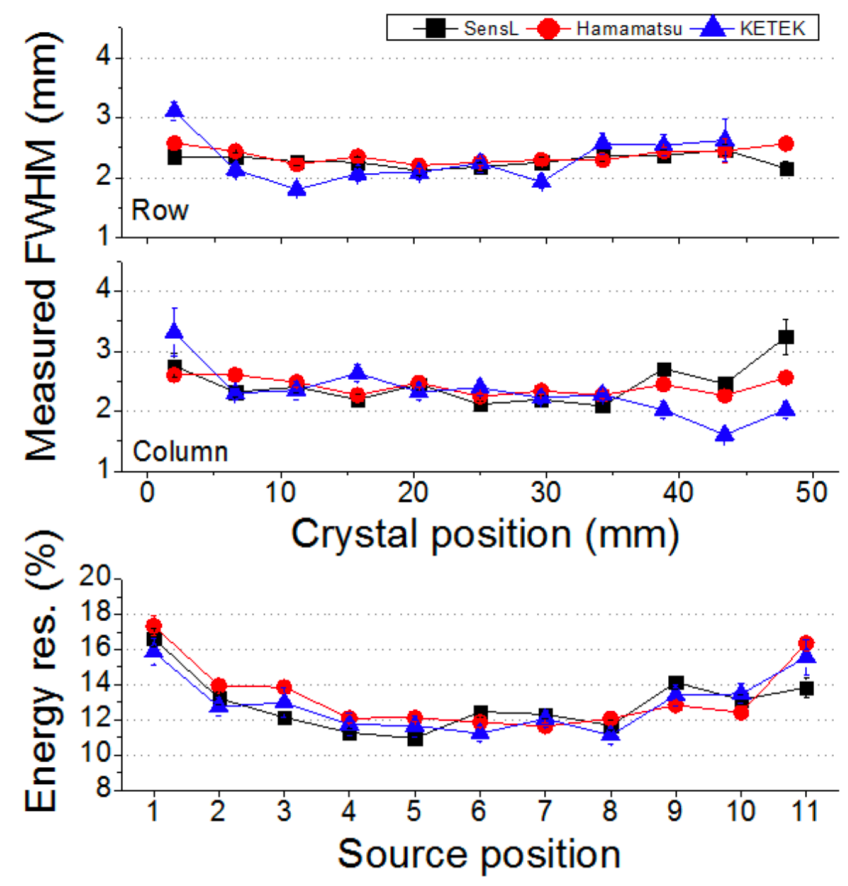

Figure 5. Top, measured spatial resolution for a row and a column of each SiPM array. Bottom, energy resolution for each source along the diagonal.

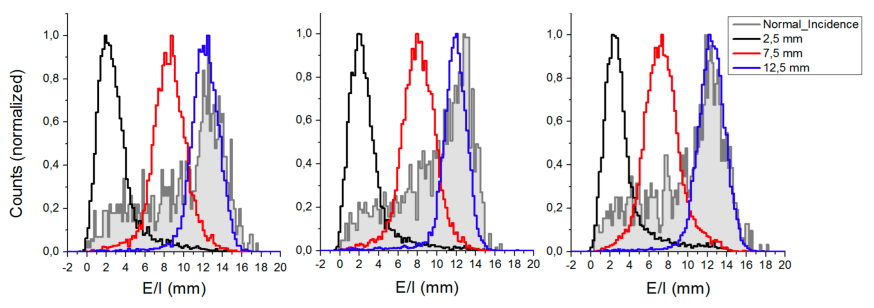

Figure 6. Measured DOI histograms for the three beam positions and for the three SiPM arrays. The overlapping distribution in grey colour was obtained when measuring in normal incidence at the same position.

Given the accurate DOI resolution we have analysed the measured data as a function of DOI layers. In particular, we have plotted the measured spatial and energy resolutions versus the DOI layer (Figure 7). DOI1 includes impacts in the $5 \mathrm{~mm}$ entrance region $(15 \mathrm{~mm}$ to $10 \mathrm{~mm}$ from photosensor), DOI2 is the central layer, and DOI3 accounts for events in the $5 \mathrm{~mm}$ closets to the photosensor array.

\subsection{Pixelated based detectors}

The determined optimum bias voltages were found to be about 2-3 V lower when compared to those found using the monolithic scintillator (see Discussion section). Table 4 shows the scanned voltage ranges. Higher voltages were not possible due to saturation effects observed in the image or energy spectra (loss of linearity). 

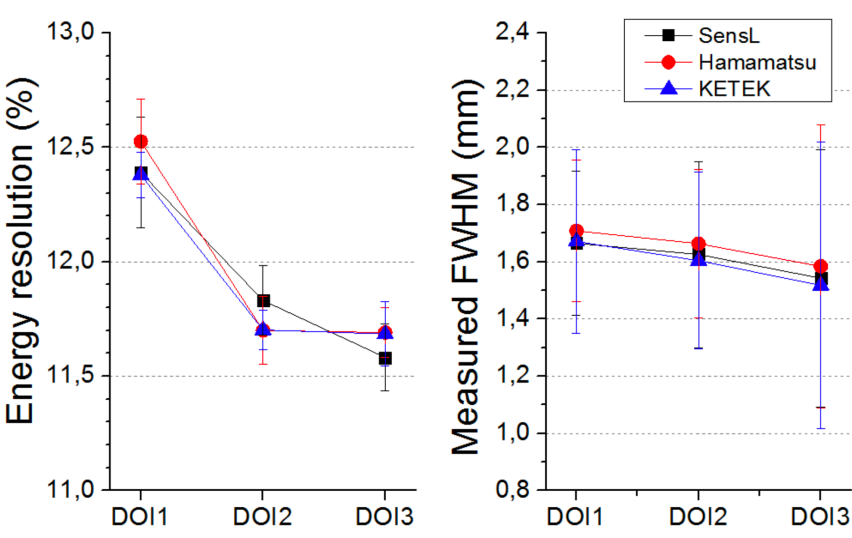

Figure 7. Measured energy resolution (left) and measured spatial resolution (right) as a function of the DOI layer.

\begin{tabular}{c|c} 
& Scanning range $(\mathrm{V})$ \\
\hline SensL & $26-27$ \\
\hline Hamamatsu & $53-54$ \\
\hline KETEK & $29-31$
\end{tabular}

Table 4. Scanning voltage range for the pixelated configuration selected for each tested SiPM array.

top shows the flood maps and bottom one the profiles of the central row of pixels for each case. Best average values of $10.5 \pm 1.9,13.4 \pm 2.2$ and $11.7 \pm 2.0$ were obtained at a bias voltage of $27 \mathrm{~V}, 54 \mathrm{~V}$ and $30 \mathrm{~V}$ for the SensL, Hamamatsu and KETEK arrays, respectively. Table 5 summarizes the average $\mathrm{P} / \mathrm{V}$ values.

\begin{tabular}{c|c|c} 
& $\mathrm{V}_{\text {bias }}(\mathrm{V})$ & $\mathrm{P} / \mathrm{V}_{\text {average }}$ \\
\hline \multirow{2}{*}{ SensL } & 26 & $10.2 \pm 1.7$ \\
\cline { 2 - 3 } & 27 & $10.5 \pm 1.9$ \\
\hline \multirow{3}{*}{ KETEK } & 29 & $10.1 \pm 1.7$ \\
\cline { 2 - 3 } & 30 & $11.7 \pm 2.0$ \\
\cline { 2 - 3 } & 31 & $11.4 \pm 1.4$ \\
\hline \multirow{2}{*}{ Hamamatsu } & 53 & $10.8 \pm 2.1$ \\
\cline { 2 - 3 } & 54 & $13.4 \pm 2.2$
\end{tabular}

Table 5. Average $\mathrm{P} / \mathrm{V}$ values for the three cases measured as a function of the applied voltage.

Regarding pixel identification, $32 \times 32$ pixel elements were distinguished in the SensL configuration, while $33 \times 33$ were identified in the KETEK and Hamamatsu cases.

Similar results are obtained for the energy resolution in all tested cases, with an average of $12 \%$ and $0.8 \%$ sigma (Figure 9). It should be mentioned that the cases named KETEK 31 $\mathrm{V}$ and Hamamatsu $54 \mathrm{~V}$, suffered from certain linearity loss, most likely overestimating the energy resolution findings.
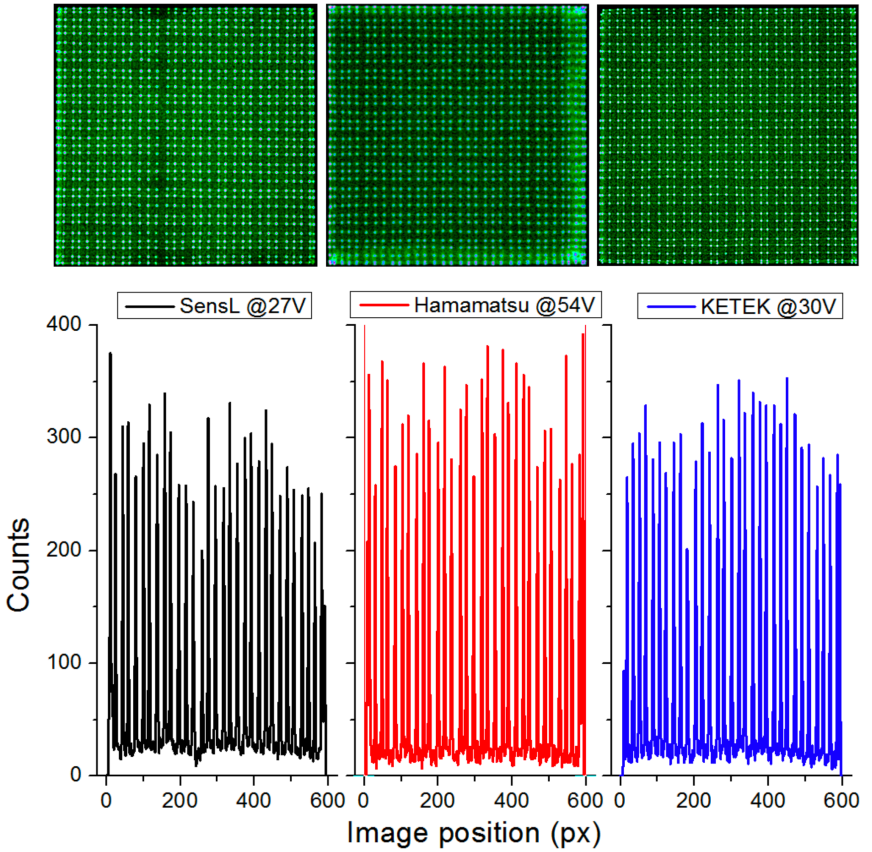

Figure 8. Flood maps of the pixelated crystal arrays. From left to right, images obtained using SensL, Hamamatsu and KETEK photosensors, respectively. The bottom plots depict the pixels profiles across the central row of pixels.

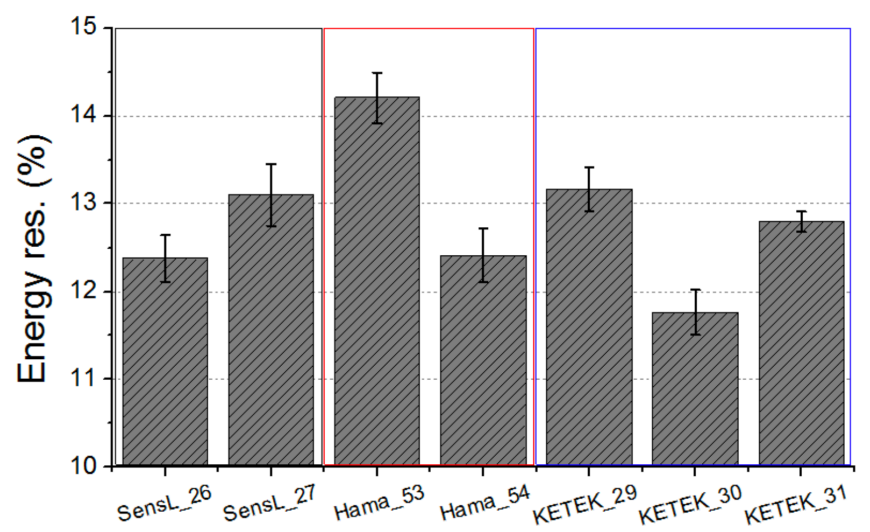

Figure 9. Energy resolution of the central pixel evaluated as a function of the $\mathrm{V}_{\text {bias }}$ for the three mentioned cases.

\section{Discussion}

In this work we have made use of three SiPM arrays with comparable specifications. SensL arrays could be obtained directly via the manufacturer whereas Hamamatsu and KETEK were explicitly built. The SiPMs had different packages but similar Silicon-based performance. The package difference should not have affected the presented results. They could be mounted keeping a similar pitch, and this reinforces again the comparable obtained results. The observed detector performance is almost independent of the SiPM arrays used in these tests.

The bias dependency of the three arrangements follows the same tendency with improved values in terms of energy 
resolution at about 4-5 $\mathrm{V}$ over the breakdown voltage. We report the most significant differences, although small in values, regarding the performance dependency of the temperature. The smallest variations were observed for SensL devices, whereas Hamamatsu parts exhibited the largest gain differences, as expected when looking to their specifications. Regarding the energy resolution performance dependency of the temperature, a slightly larger degradation was found for KETEK devices, most likely due to the reported higher dark count rates. The obtained results are in good agreement with the values provided by each manufacturer (Table 1).

A somehow more detailed analysis was carried out both using normal and lateral incidence experiments to detector blocks, when the SiPM arrays were coupled to thick blackpainted (including the RR layer) monolithic scintillation crystals. The same reference detector, associated electronics and scintillator was used in all measurements. Once more, the performance of the three tested detector blocks (SiPM arrays combined) was comparable, in terms of energy, measured spatial and DOI resolutions. When the data were split into DOI layers, energy and measured spatial resolution behaved as expected, improving closer to the photosensor, and exhibiting similar values.

In experiments with the pixelated crystal array of $1.5 \mathrm{~mm}$ pitch, we found an optimum bias voltage slightly lower (about $2 \mathrm{~V}$ ) than in the case of using a continuous crystal. This is produced because the collection of scintillation light by the photosensors increases in the pixelated case due to their smaller geometry and type of treatment to the surfaces, making it possible to collect larger amounts of scintillation light in one SiPM. The frontend electronics and DAQ system utilized in these set-ups are somehow tuned for lower scintillation light collections, as typically observed when using monolithic crystals, and this induced the different optimum bias.

When the photosensors were coupled to pixelated arrays, we found always possible to resolve most of $33 \times 33$ elements, independently of the SiPM array. The difference in the number of identified pixels might have occurred due to some miss-coupling between the crystal and the photosensor in the SensL case. The determined peak-to-valley values were also similar for all cases. Slightly better energy resolutions than in the case of the continuous crystal were observed, due to the better scintillation light collection.

Regarding timing resolution, we have used electronics with timing resolutions in the 1.5-2 ns range and, thus, not explicitly studied. Other works have shown that SiPMs of these particular manufactures exhibit similar timing performances [25]-[27].

\section{Conclusion}

We provide in this work, for the first time, a comparable study of three SiPM arrays of $12 \times 12$ elements each with similar geometry, covering about $5 \mathrm{~cm} \times 5 \mathrm{~cm}$. We have studied their performance as a function of the temperature and supplied bias voltage, as well as when coupled to crystal arrays or monolithic blocks. Some studies with smaller SiPM arrays and pixelated crystal have carried out before, but not a detail comparison study also with monolithic blocks and large area SiPM arrays. We can conclude that the three SiPMs perform almost identical in the typical conditions of gamma ray detectors suitable for gamma cameras, SPECT or PET applications, among others.

\section{Acknowledgements}

This project has received funding from the European Research Council (ERC) under the European Union's Horizon 2020 research and innovation program (grant agreement No 695536). It has also been supported by the Spanish Ministerio de Economía, Industria y Competitividad under Grant TEC2016-79884-C2-1-R.

\section{References}

[1] D. Henseler, et al., "SiPM performance in PET applications: An experimental and theoretical analysis," Proc. IEEE NSSMIC, Orlando, USA, 2009.

[2] E. Roncali and S.R. Cherry, "Application of silicon photomultipliers to positron emission tomography," Ann. Biomed. Eng. 39, 1358-1377, 2011.

[3] V.D. Kovaltchouk, et al., "Comparison of a silicon photomultiplier to a traditional vacuum photomultiplier," Nucl. Instrum. Methods A. 538, 1-3, 408-415, 2005.

[4] B. Sanaei, et al., "Characterization of a New Silicon Photomultiplier in Comparison with a Conventional Photomultiplier Tube,” Int. J. Mod. Phys. A. 6, 425-433, 2015.

[5] P. Buzhan, et al., "Silicon photomultiplier and its possible applications," Nucl. Instrum. Methods A. 504, 1-3, 48-52, 2003.

[6] G. Barbarino, et al., "Silicon Photo Multipliers Detectors Operating in Geiger Regime: an Unlimited Device for Future Applications," IntechOpen. Available from: https://www.intechopen.com/books/photodiodes-worldactivities-in-2011/silicon-photo-multipliers-detectorsoperating-in-geiger-regime-an-unlimited-device-for-futureapplic.

[7] B. Dolgoshein, et al., "Status report on Silicon Photomultiplier development and its applications," Nucl. Instrum. Methods A. 563, 368-376, 2006.

[8] R. Rando, et al., "Silicon Photomultiplier Research and Development Studies for the Large Size Telescope of the Cherenkov Telescope Array," PoS ICRC2015 940, 2016.

[9] P. Bérard, et al., "Performance Measurement for a New Low Dark Count UV-SiPM," Proc. IEEE NSS-MIC, Valencia, Spain, 2011. 
[10] A.J. Gonzalez, et al., "Initial Results of the MINDView PET Insert Inside the 3T mMR," IEEE Trans. Radiat. Plasma Med. Sci., in press, doi: 10.1109/TRPMS.2018.2866899, 2018.

[11] L. Moliner, et al., "Design and evaluation of the MAMMI dedicated breast PET," Med. Phys. 39, 5393, 2012.

[12] F. Sanchez, et al., "ALBIRA: A small animal PET/SPECT/CT imaging system," Med. Phys. 40, 051906-1, 2013.

[13] A.J. Gonzalez, et al., "A PET Design Based on SiPM and Monolithic LYSO Crystals: Performance Evaluation,” IEEE Trans. Nucl. Sci. 63, 2471, 2016.

[14] https://sensl.com

[15] https://www.hamamatsu.com/

[16] https://www.ketek.net/sipm/

[17] SensL light, "C-Series Low noise, Blue-Sensitive Silicon Photomultipiers," Datasheet, 2014.

[18] HAMAMATSU Photonics, "MPPC (Multi-Pixel Photon Counter), S13360 series," Datasheet, 2016.

[19] KETEK GmbH, "SiPM-Silicon Photomultiplier PM3315WB/PM3325-WB," Datasheet, 2018.

[20] A. Gonzalez-Montoro, et al., "Performance Study of a Large Monolithic LYSO PET Detector With Accurate Photon DOI Using Retroreflector Layers," IEEE Trans. Radiat. Plasma Med. Sci. 1, 229-237, 2017.

[21] Saint-Gobain, "BC-630 Silicone Grease," Datasheet, 2017.

[22] A. J. González, et al., "The MINDView brain PET detector, feasibility study based on SiPM arrays," Nucl. Instrum. Methods A 818, 82-90, 2016.

[23] R. Pani et al., "Preliminary evaluation of a monolithic detector module for integrated PET/MRI scanner with high spatial resolution," J. Instrum. 10, C06006, 2015.

[24] A. González-Montoro, et al., "Detector block performance based on a monolithic LYSO crystal using a novel signal multiplexing method," Nucl. Instrum. Methods A. 912, 372377, 2018.

[25] A. Ronzhin, et al., "A SiPM-based TOF-PET detector with high speed dgital DRS4 readout," Nucl. Instrum. Methods A 703, 109-113, 2013.

[26] C. Kim, et al., "Time-of-flight PET-MR detector development with silicon photomultipliers," Proc. IEEE NSS-MIC, Anaheim, USA, 2012.

[27] D. R. Schaart, et al., "A novel SiPM-array-based, monolithic scintillator detector for PET," Phys. Med. Biol. 54, 11, 35013512, 2009. 